\title{
BMJ Open Diagnosis, treatment and management of apathy in Parkinson's disease: a scoping review
}

\author{
Bria Mele (D) , , ${ }^{1,2}$ Shinia Van, ${ }^{2}$ Jayna Holroyd-Leduc, ${ }^{1,3,4}$ Zahinoor Ismail, , ,2,5,6,7,8 \\ Tamara Pringsheim (D) , ${ }^{1,6,7}$ Zahra Goodarzi ${ }^{1,4,7,8}$
}

To cite: Mele B, Van S, HolroydLeduc J, et al. Diagnosis, treatment and management of apathy in Parkinson's disease: a scoping review. BMJ Open 2020;10:e037632. doi:10.1136/ bmjopen-2020-037632

- Prepublication history and additional material for this paper are available online. To view these files, please visit the journal online (http://dx.doi. org/10.1136/bmjopen-2020037632).

Received 10 February 2020 Revised 14 May 2020 Accepted 19 July 2020
Check for updates

(C) Author(s) (or their employer(s)) 2020. Re-use permitted under CC BY-NC. No commercial re-use. See rights and permissions. Published by BMJ.

For numbered affiliations see end of article.

Correspondence to

Bria Mele;

bria.mele@ucalgary.ca

\section{ABSTRACT}

Objective To conduct a scoping review of the literature on apathy in Parkinson's disease (PD), to better understand how apathy in Parkinson's disease is diagnosed, treated and managed.

Methods MEDLINE, Embase, PsycINFO, CINAHL, Cochrane Central Register of Control Trials and Cochrane Database of Systematic Reviews were searched to 17 May 2017. An updated review was run from 17 May 2017 to 28 January 2019. The grey literature was searched using the CADTH Grey Matters tool. Original peer-reviewed research was included if it included individuals with PD and apathy. Non-original data was only included if it was in the form of meta-analysis. All information regarding diagnosis, treatment and management of PD was extracted. Citation screening and extraction were performed in duplicate. Results From 11375 citations, 362 articles were included in the final review. The majority of included studies focussed on prevalence, with few studies examining treatment. Twenty screening tools for apathy were identified. Fifty per cent of treatment studies were randomised control trials (RCTs). RCTs applied treatment methods including: exercise, mindfulness, rotigotine (Neupro) transdermal patch and rivastigmine (Exelon). Conclusions This review identified a large body of literature describing current knowledge on diagnosing, treating and managing apathy in PD. Future research should aim to detect an ideal screening tool for apathy in PD, to identify the best treatment options for apathy and the variety of comorbidities it may present with and finally aim to better understand postoperative apathy in those with deep brain stimulation.

\section{INTRODUCTION}

Persons with apathy typically present with behavioural, emotional and cognitive impairment including reduced interest, reduced initiative and motivation, emotional distress and intellectual impairment. ${ }^{1-3}$ Apathy is present in a number of neurodegenerative diseases, including Huntington's disease, multiple sclerosis, Alzheimer's disease (AD) and Parkinson's disease (PD). ${ }^{4}$ Apathy is present in $17 \%$ to $70 \%$ of those with PD. ${ }^{56}$ This wide range of prevalence estimates demonstrates the heterogeneity of how apathy and

\section{Strengths and limitations of this study}

Our scoping review is the first synthesis of all peerreviewed literature on apathy in Parkinson's disease (PD) and applied the Peer Reviewed Electronic Search Strategy to ensure we used a comprehensive search strategy.

- Our scoping review provides a comprehensive assessment of diagnostic and treatment literature on apathy in PD, which can inform current practice as well as future research given gaps identified.

- Limitations of this research include the potential that we have missed important original literature on apathy in PD, we also did not include non-peer-reviewed literature.

- We did not conduct a formal quality assessment of included studies.

associated neuropsychiatric comorbidities are defined within the literature. In PD, apathy results in poor response to motor symptom treatment, increased healthcare spending, decreased quality of life for persons with PD and their caregivers, as well as increased risk of developing dementia and increased difficulty making decisions in day-to-day life. ${ }^{7-12}$

The current lack of consensus regarding a used definition of apathy limits the diagnostic approach taken to identify apathy in PD. There are a variety of screening tools to identify the presence and severity of apathy in both PD and neurodegenerative diseases in general. ${ }^{13}$ A key limitation of these tools is the lack of standard criteria with which the tool validity can be assessed. ${ }^{13} 14$ A recent systematic review of apathy screening tools concluded the Apathy Scale (AS) is clinically valid when looking to exclude the presence of apathy symptoms to assess medication side effects. ${ }^{15}$ The Lille Apathy Rating Scale (LARS) was also reported as clinically valid for diagnosing apathy. ${ }^{15}$

In many cases, improvements in apathy are a secondary outcome to treating other neuropsychiatric symptoms in PD. ${ }^{16-20}$ For example, 
treatment methods, such as cholinesterase inhibitors may improve apathy as a secondary outcome to improvements in cognitive impairment. ${ }^{21}$ This highlights the need for research looking at apathy isolated from other symptoms that may present in those with PD, to better understand how to treat solely apathy.

While it has been argued that apathy may be a part of a number of other symptoms present in neurodegenerative diseases, including depression and cognitive impairment, ${ }^{14}$ 22-24 numerous studies have identified the underlying mechanisms of these symptoms to be separate. ${ }^{22} 23$ 25-28 This understanding emphasises the importance of synthesising the apathy literature to better understand apathy as an isolated symptom; in order to direct future development of validated screening tools and treatment approaches for apathy.

A systematic review assessing the prevalence of apathy in PD reported a prevalence of $40 \% .{ }^{6}$ Authors identified that the variation in apathy and depression definitions used, as well as whether cognitive function was reported, as significant contributors to heterogeneity (I2=93\%). While the authors stratified their pooled-prevalence analysis by the variety of available apathy definitions, presence of confounding depression and cognitive function, heterogeneity remained significant. ${ }^{6}$

To address gaps in the apathy literature, we aimed to conduct a scoping review to assess conceptual boundaries and working definitions of apathy ${ }^{29}$ Specifically, the purpose of this scoping review was to synthesise all available literature regarding the diagnosis, treatment and management of apathy in PD and assess gaps in knowledge regarding understanding an optimal approach to apathy in PD. This will provide a clear map of the evidence and clearly define evidence gaps to guide future research.

\section{METHODS}

The objectives, inclusion criteria and methods for this scoping review were prespecified and published in a protocol with Open Science Framework. ${ }^{30} 31$ We used previously established scoping review methodology to guide our study methods and applied the Preferred Reporting Items for Systematic Reviews and Meta-Analyses for Scoping Reviews (PRISMA-Scr). ${ }^{32-34}$

\section{Search strategy and literature sources}

The databases searched were MEDLINE, Embase, PsycINFO, CINAHL, Cochrane Central Register of Control Trials and Cochrane Database of Systematic Reviews. These databases were searched from inception to 17 May 2017. An updated search was conducted from 17 May 2017 to 29 January 2019. There were two main search clusters: PD and apathy. The Medical Subject Headings, Emtree, PsycINFO and Cochrane terms were combined within each cluster using 'or', these clusters were then combined using 'and' (online supplementary table 1 and 2). Given the overlap of anhedonia with both apathy and depression, we did not include anhedonia as a final search term. The final search strategy was developed with two experienced research librarians, as per the Peer Review for Electronic Search Strategies. ${ }^{35}$ The grey literature was searched using the CADTH Grey Matters tools in tandem with searches of all relevant websites associated with PD and non-motor symptoms (NMS) in movement disorders. ${ }^{36}$

\section{Inclusion criteria}

Inclusion criteria were defined based on the Patient, Concept, Context criteria as per the Joanna Briggs Institute methodology for scoping reviews: any papers directly discussing apathy in PD and diagnosis, treatment or management were included. ${ }^{29}$ As per Effective Practice and Organisation of Care reviews criteria the following study designs were considered for inclusion: randomised control trials, non-randomised control trials, controlled before-after studies and interrupted time series. Case reports and case series were also considered for inclusion. If non-English publications presented with an English language abstract with sufficient evidence for extraction, they were included. Non-original data was only included if it was in the form of meta-analysis. All other nonoriginal articles, grey literature and included articles had their references hand searched to ensure their references had already been included. The abstract stage inclusion criteria were any study that discussed apathy in PD, at the full-text stage the study had to include information on diagnosis, treatment or overall management of PD in persons with $\mathrm{PD}$, that could be applied within the context of a movement disorders clinic. All abstracts and full-texts from the original search were reviewed individually by two authors (BSM and ZSG). All abstracts and full-texts from the updated search were reviewed individually by two authors (BSM and SV). At the abstract stage disagreement was resolved through inclusion, at the full-text stage a third party (JH-L or ZSG) was brought in for discussion if required. Agreement between the two authors was assessed during the full-text, using the kappa statistic.

\section{Data extraction, synthesis and analysis}

A charting table was developed a priori by the two reviewers (BSM and ZSG). The charting table consisted of predefined data items for extraction, which would inform understandings of what is currently known within the literature regarding diagnosis and treatment of apathy in $\mathrm{PD}$, as well as demographic information regarding patient and study characteristics. Where appropriate, numerical data was extracted to provide descriptive statistical analyses of the included studies. Included articles were sorted based on type of information provided: diagnostic, treatment, post deep-brain stimulation (DBS) apathy, quality of life, caregiver or meta-analyses. This framed the type of information extracted during the charting process. The charting form was revised iteratively, as needed while screening the first 50 articles. One reviewer (BSM) extracted data from the original search; the second reviewer then independently verified the data (ZSG). 
Two authors independently extracted half of the data, and verified the other half of the data from the updated search (BSM and SV). Any discrepancies were resolved by re-review of the study or discussion with the third reviewer (JH-L).

\section{Patient and public involvement}

No patient involved.

\section{RESULTS}

The electronic database search yielded 4035 citations; when duplicates were removed, there were 3537 records screened; 1443 full-text articles were reviewed. Three hundred and twenty-one studies were included from the original search (online supplementary table 3A, cited in online supplementary table 4A). The updated search, which included studies published from 7 May 2017 to 29 January 2019 yielded 644 citations; when duplicates were removed, there were 503 records screened; 216 full-text articles were reviewed, 40 of which were included in the final synthesis (cited in online supplementary table 4A).
The grey literature search produced 7335 citations; 1 was included in the final review. The total number of studies included from both the original and updated search was 362 studies (figure 1). Screening agreement between raters at the full-text inclusion stage was $54 \cdot 4(\kappa=0 \cdot 94,95 \%$ $\mathrm{CI}=0.89$ to $1 \cdot 00)$. Screening agreement between raters at the full-text stage of the updated search was $81.4(\kappa=0 \cdot 63$, $95 \% \mathrm{CI}=0.42$ to 0.84 ). Exclusion criteria are provided in figure 1 . The most common reason for exclusion was conference abstracts when no full-text peer-reviewed paper was identified $(n=493)$. Conference abstract titles were searched for full-text papers and included if relevant; due to the lack of a formal peer-review process for conference abstracts, if no full-text peer-reviewed text was found, they were excluded.

\section{Description of included studies}

The majority of studies were published out of Europe $(56 \%)$; overall seven continents published research on apathy in PD (table 1). Studies were published between 1991 and 2017, 41\% of which were published between

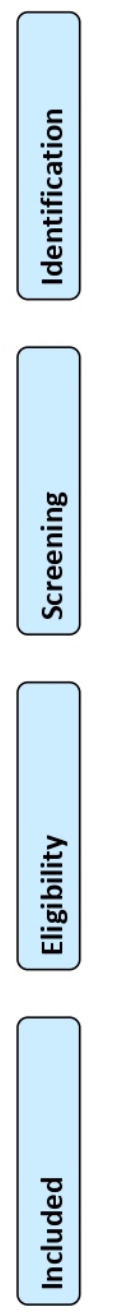

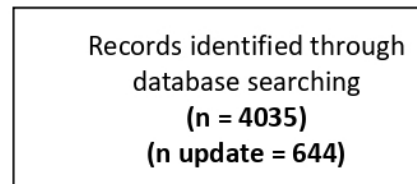

( $n=4035$ )

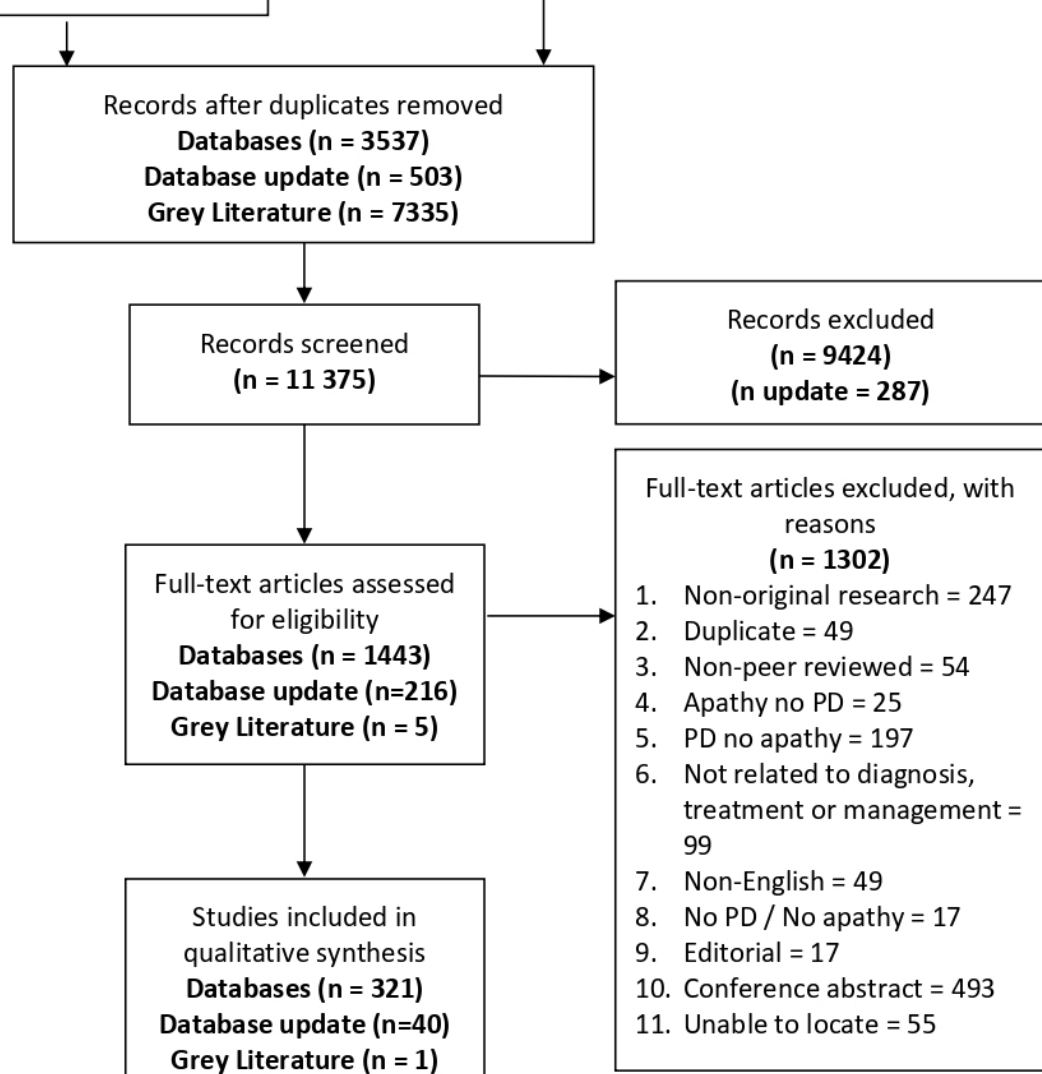

Figure 1 PRISMA flow diagram. PD, Parkinson'sdisease; PRISMA, Preferred Reporting Items for Systematic Reviews and Meta-Analyses. 
Table 1 Summary of included studies characteristics

Number (\%) of

Characteristic

362 citations

Country of origin*

Africa

$1(0.3)$

Asia

50 (13.8)

Australia

$4(1.1)$

Europe

$203(56.1)$

North America

$79(21.8)$

South and Central America

$9(2.5)$

Cross-continental

$16(4.4)$

Study design

Before-after

$62(17.1)$

Case report

$6(1.7)$

Case-control

24 (6.6)

Cohort

45 (12.4)

Cross-sectional

$198(54.7)$

Letter

$1(0.2)$

Meta-analysis

$2(0.6)$

Qualitative

$2(0.6)$

Randomised control trial

$22(6.1)$

Primary focus of article $\dagger$

Prevalence/description of NMS in PD/ 146 (40.3) epidemiological

$\begin{array}{lc}\text { Diagnostic } & 49(13.5) \\ \text { Treatment/management } & 48(13.3) \\ \text { Patient quality of life } & 26(7.2) \\ \text { Caregiver quality of life } & 5(1.4) \\ \text { Post deep brain stimulation } & 54(14.9) \\ \text { Brain imaging/mechanisms } & 28(7.7) \\ \text { Decision-making/reward } & 6(1.7)\end{array}$

Toolsł

Apathy Evaluation Scale

$71(17.3)$

Apathy Inventory

$4(1.0)$

Ardouin Scale

$1(0.2)$

Apathy Scale

$107(26.1)$

Behavioural Dysexecutive Syndrome Inventory

$1(0.2)$

Cambridge Behavioural Inventory

$1(0.2)$

Diagnostic Criteria

$4(1.0)$

Dimensional Apathy Scale

$3(0.7)$

Frontal Behavioural Inventory

$1(0.2)$

Frontal Systems Behaviour Scale

$9(2.2)$

Clinical Interview

$6(1.5)$

Lille Apathy Rating Scale

$40(9.8)$

Modified Indifference Scale

$1(0.2)$

Montgomery-Asberg Depression Scale

$1(0.2)$

Neuropsychiatric Inventory

$50(12.2)$
Table 1 Continued

Characteristic

Number (\%) of 362 citations

$\begin{array}{lr}\text { Non-motor Symptoms Questionnaire } & 8(2.0) \\ \text { Non-Motor Symptoms Scale } & 26(6.3) \\ \begin{array}{l}\text { Parkinson's Disease Dementia Short } \\ \text { Screen }\end{array} & 1(0.2) \\ \begin{array}{l}\text { Scale for apathy in institutionalised } \\ \text { persons with dementia }\end{array} & 1(0.2) \\ \begin{array}{l}\text { Scale for Evaluations of } \\ \text { Neuropsychiatric Disorders in PD }\end{array} & 4(1.0) \\ \begin{array}{l}\text { Snaith Pleasure Scale } \\ \text { Symptom Checklist 90 Revised }\end{array} & 2(0.5) \\ \begin{array}{l}\text { Unclear } \\ \text { Unified Parkinson's Disease Rating } \\ \text { Scale } \\ \text { Visual Analogue Scale }\end{array} & 1(0.2) \\ \end{array}$

*African countries included: Nigeria; Asian countries included: China, India, Israel, Japan, Korea, Malaysia, Russia, Saudi Arabia, Singapore and Taiwan; Australian countries included: Australia and New Zealand; European countries included: Austria, Belgium, Finland, France, Germany, Hungary, Italy, Netherlands, Norway, Poland, Portugal, Romania, Serbia, Slovakia, Spain, Sweden, Switzerland, Turkey and UK; North American countries included: Canada, Mexico and the USA; South and Central American countries included: Argentina, Brazil and Cuba.

†Many studies had more than one objective, but were categorised by their primary objective.

$\ddagger$ A tool was employed a total of 410 times, with some studies employing more than one tool.

NMS, non-motor symptoms; PD, Parkinson's disease.

2014 and 2017 (figure 2). The primary focus of 147 articles was assessing prevalence or characteristics of general non-motor symptoms including apathy and their presentation in PD. These studies were considered general or epidemiological studies (table 1). The most common study designs were cross-sectional studies (55\%) in many cases nested within cohort studies, followed by beforeafter studies (17\%) (table 1). To ensure we could provide a complete overview of all information on apathy in PD and as per scoping review recommendations, we did not conduct a formal assessment of methodological quality and bias. ${ }^{3738}$

\section{Description of diagnostic information}

Forty-nine studies explicitly discussed the diagnosis of apathy in PD (14\%) (table 1; online supplementary table 3A). In 2009, experts on PD from around the world proposed the following criteria for diagnosing apathy in PD: Loss of or reduced: motivation, goal-directed behaviour, goal-directed cognitive activity, emotion, with at least one of the latter three criteria present for at least 4 weeks for the majority of that time. ${ }^{39}$ However, these criteria were referred to as the gold standard in only two included studies. ${ }^{40}$ No other formalised definitions, outside what was proposed by an apathy-screening tool, 


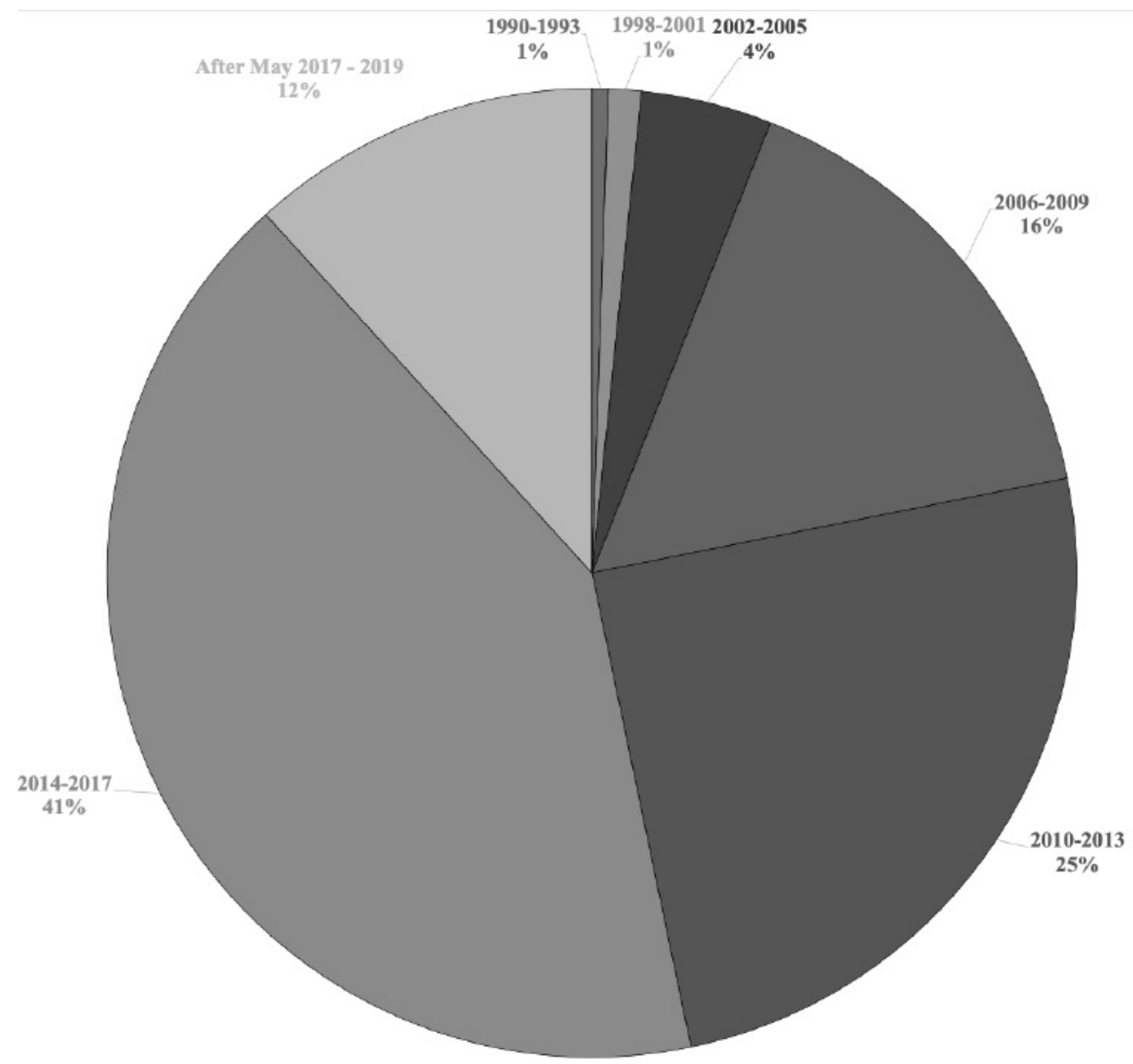

Figure 2 Summary of publication years of included studies.

were used in the other included studies. Where studies also included an assessment of common comorbidities of apathy, such as depression and cognitive impairment, the influence these comorbidities may have had on apathy measures was not consistently reported.

Fourteen tools screened for apathy as part of a larger non-motor symptom-screening tool. The most commonly used of these tools was the Neuropsychiatric Inventory (NPI) used by $12 \%$ of studies or Neuropsychiatric Inventory Questionnaire (NPI-Q), used by $2 \%$ of these studies. The NPI was originally developed to assess 10 common behavioural symptoms in dementia populations, including apathy. ${ }^{42}$ The NPI-Q is a shortened version of the original NPI. ${ }^{43}$ Tool questions are administered to caregivers instead of the persons with the neurological disease. ${ }^{42}$ The NPI is copyrighted, however, it is free to use for investigators researching through an academic institution. The Movement Disorder Society - Unified Parkinson's Disease Rating Scale (UPDRS), specifically Part 1, Item 4 was the second most common non-apathy specific tool used $(10 \%) .^{44}$ This tool was developed to assess both motor and non-motor symptoms in PD. ${ }^{44}$ This tool is copyrighted, and permission must be granted by the Movement Disorder Society to employ the tool; training programmes for the tool are also provided through the Movement Disorder Society.
Six tools were apathy-specific screening tools; the AS was most frequently applied $(26 \%) .{ }^{45}$ This tool was specifically designed for assessing the severity of apathy in PD populations. ${ }^{45}$ The second most common apathy specific tool employed (17\%) was the Apathy Evaluation Scale (AES).$^{46}$ This tool was originally validated for use in populations with stroke, $\mathrm{AD}$, major depression and elderly populations. ${ }^{46}$ Both AS and AES tools are not copyrighted and are easily accessible online.

A variety of studies assessed differences between self versus caregiver rated versions of non-motor symptom screening tools. ${ }^{4-50}$ It was reported that where a screening tool is administered to the caregiver, apathy scores are higher; this is thought to be due to caregivers increased awareness of the presence and severity of apathy in those they are caring for. ${ }^{47-50}$ Two per cent of studies that did not employ any screening tools used clinical interviews to identify apathy.

\section{Description of treatment information}

Forty-eight studies explicitly discussed treatment (table 1; table 2; online supplementary table 3A), of which 22 were randomised control trials (RCT) $(46 \%)$. Ten studies had the primary objective of improving apathy or motivation in PD specifically. ${ }^{16} 1751-58$ One of these studies was a meta-analysis assessing the overall effect of pramipexole 
Table 2 Summary of treatment types

\begin{tabular}{|c|c|c|c|}
\hline & Treatment type & $\begin{array}{l}\text { Number }(\%) \text { of } \\
48 \text { citations }\end{array}$ & $\begin{array}{l}\text { Study design and sample size (N) (reference } \\
\text { number*) }\end{array}$ \\
\hline \multirow[t]{27}{*}{ Pharmacotherapies } & Apomorphine & $1(2.1)$ & Before-after (18) (21) \\
\hline & $\begin{array}{l}\text { Apomorphine and rotigotine } \\
\text { transdermal patch }\end{array}$ & $1(2.1)$ & Before-after (15) (296) \\
\hline & Docosahexaenoic acid & $1(2.1)$ & Randomised control trial (24) (234) \\
\hline & \multirow[t]{4}{*}{ Dopamine therapies } & \multirow[t]{4}{*}{$4(8.3)$} & Before-after (53) (100) \\
\hline & & & Before-after (11) (156) \\
\hline & & & Cross-sectional (199) (158) \\
\hline & & & Cross-sectional (515) (227) \\
\hline & Istradefylline & $1(2.1)$ & Non-randomised control trial (30) (17) 4A \\
\hline & Tianeptine & $1(2.1)$ & Before-after (17) (152) \\
\hline & Galantamine & $1(2.1)$ & Randomised control trial (41) (159) \\
\hline & MAO inhibitor and levodopa & $1(2.1)$ & Cross-sectional (76) (140) \\
\hline & Methylphenidate & $1(2.1)$ & Case study (1) (47) \\
\hline & Milnacipran & $1(2.1)$ & Before-after (8) (178) \\
\hline & Atomoxetine & $1(2.1)$ & Randomised control trial (55) (307) \\
\hline & Pramipexole & $1(2.1)$ & Meta-analysis (NR) (147) \\
\hline & $\begin{array}{l}\text { Pramipexole and } \\
\text { methylphenidate }\end{array}$ & $1(2.1)$ & Randomised control trial (46) (77) \\
\hline & Rasagiline & $2(4.2)$ & $\begin{array}{l}\text { Randomised control trial (123) (25) } \\
\text { Randomised control trial (191) (279) }\end{array}$ \\
\hline & \multirow[t]{4}{*}{ Rivastigmine } & \multirow[t]{4}{*}{$4(8.3)$} & Case report (1) (36) \\
\hline & & & Before-after (48) (193) \\
\hline & & & Before-after (23) (207) \\
\hline & & & Randomised control trial (30) (72) \\
\hline & \multirow[t]{4}{*}{ Rotigotine (transdermal patch) } & \multirow[t]{4}{*}{$4(8.3)$} & Randomised control trial (349) (17) \\
\hline & & & Randomised control trial (267) (49) \\
\hline & & & Randomised control trial (380) (54) \\
\hline & & & Randomised control trial (122) (115) \\
\hline & Serotonergic therapies & $2(4.2)$ & $\begin{array}{l}\text { Cross-sectional (45) (167) } \\
\text { Observational cohort (181) (314) }\end{array}$ \\
\hline & Yokukansan & $1(2.1)$ & Before-after (25) (113) \\
\hline \multirow[t]{10}{*}{ Non-pharmacotherapies } & Behavioural planning & $1(2.1)$ & Before-after (34) (38) \\
\hline & $\begin{array}{l}\text { Chronic disease self- } \\
\text { management and exercise }\end{array}$ & $1(2.1)$ & Randomised control trial (30) (27) 4A \\
\hline & Cognitive training programme & $1(2.1)$ & Randomised control trial (42) (225) \\
\hline & Dance & $2(4.2)$ & $\begin{array}{l}\text { Randomised control trial (46) (111) } \\
\text { Randomised control trial (40) (255) }\end{array}$ \\
\hline & Equine-assisted interventions & $1(2.1)$ & Case study (3) (21) 4A \\
\hline & \multirow[t]{3}{*}{ Exercise } & \multirow[t]{3}{*}{$3(6.3)$} & Qualitative study (8) (204) \\
\hline & & & Randomised control trial (58) (133) \\
\hline & & & Randomised control trial (20) (62) \\
\hline & Mindfulness & $1(2.1)$ & Before-after (39) (44) \\
\hline & Psycho-education & $1(2.1)$ & Randomised control trial (19) (266) \\
\hline
\end{tabular}




\begin{tabular}{llll}
\hline & Treatment type & $\begin{array}{l}\text { Number (\%) of } \\
\mathbf{4 8} \text { citations }\end{array}$ & $\begin{array}{l}\text { Study design and sample size (N) (reference } \\
\text { number*) }\end{array}$ \\
\hline Surgical & $\begin{array}{l}\text { Bilateral subthalamotomies } \\
\text { Deep brain stimulation } \\
\text { subthalamic nucleus }\end{array}$ & $1(2.1)$ & Before-after (10) (27) \\
& $6(12.5)$ & Randomised control trial (56) (213) & Randomised control trial (12) (117) \\
& & Randomised control trial (15) (185) \\
& Randomised control trial (63) (294) \\
& Ventrolateralis thalamotomy & $1(2.1)$ & Randomised control trial (123) (309) \\
\end{tabular}

MAO, monoamine oxidase.

on mood and motivation in PD. ${ }^{58}$ While measures of motivation within the included studies were not considered a sufficient diagnosis of apathy by the authors, it is important to note an overall decrease in motivational deficit score on the UPDRS Part 1 Item 4 was observed with pramipexole. ${ }^{58}$ The remaining studies were primarily concerned with treating depression, cognitive impairment or other non-motor symptoms, assessing improvements in apathy only as a secondary outcome.

Twenty-nine studies $(60 \%)$ employed a pharmacological intervention (table 2). Rivastigmine (an acetylcholinesterase inhibitor typically used to treat dementia) ${ }^{16-19}$ and rotigotine (a dopamine agonist commonly used to treat the movement symptoms in PD) $)^{52} 545960$ were two of the most commonly prescribed interventions. In a randomised control trial with 122 participants, the rotigotine (Neupro) transdermal patch appeared to decrease apathy in those with PD and no dementia. ${ }^{54}$ Rivastigmine (Exelon) was found to decrease apathy and caregiver burden due to apathy. ${ }^{1618} 19$ Two of the rivastigmine studies were non-randomised control trials with 48 and 23 participants, respectively. ${ }^{17} 19$ While the other two studies were a randomised control trial with 30 participants and a case study with one participant. ${ }^{16} 18$ One study found no prolonged benefit of rivastigmine. ${ }^{17}$ Comparably, galantamine (Razadyne) typically used to treat dementia, reduced apathy in a non-randomised control trial with 41 participants with PD dementia. ${ }^{20}$

Remaining studies used non-pharmacological interventions including exercise, behavioural therapy and brain stimulation. Notably, apathy was improved in four of the five exercise-based interventions; exercise programmes included Nordic walking, and scheduled exercise routines (e-62, e-111, e-133). Three of the four studies considered an improvement of apathy as a statistically significant decrease in apathy as measured by the AS or LARS (e-62, e-111, e-133). These three studies were all randomised control trials with 20, 46 and 58 participants, respectively (e-62, e-111, e-133). The fourth study applied qualitative methods among eight participants, and found patients reported using exercise as a means to overcome apathy (e-204). The fifth exercise based randomised control trial, with 40 participants study purports their population was underpowered to identify a statistically significant decrease in AS scores (e-255). A non-statistically significant decrease in AS scores was reported in one observational cohort study with 39 participants, where mindfulness training was used, which applied a combination of mediation, writing and yoga (e-44).

\section{Postoperative apathy in $\mathrm{PD} /$ mechanisms of apathy in PD}

Any study that described apathy that occurred post-DBS, was considered non-de novo apathy and categorised separately from above studies, which are concerned with de novo apathy. Fifty-four studies discussed post-DBS apathy (table 2; online supplementary table 3A). The most common objective of these studies was assessing changes in mood following surgery. Specifically, the role of dopamine medication withdrawal was investigated as a key mechanism to explain the onset of apathy post-surgery (e-63, e-64). Overall, results were inconclusive, with no clear association between increased apathy and DBS.

Management approaches taken in those with de novo apathy versus apathy occurring post-DBS also differed. It was hypothesised that apathy onset after DBS is due to withdrawal from dopamine medications post-surgery (e-63, e-64). The mechanisms of de novo apathy, discussed below, are not as clearly understood. It was reported that dopamine replacement therapy improved apathy induced by DBS but was not found to be effective in de novo PD (e-63, e-64, e-295).

The specific mechanisms of apathy were assessed in 28 studies (online supplementary table $3 \mathrm{~A}$ ). The most commonly proposed mechanisms to explain de novo apathy was dysfunction of the basal ganglia system, specifically within the posterior and anterior cingulate cortex, nucleus accumbens, striatum, subthalamic nucleus. This system is also implicated to play a role in cognitive function, which may explain why cognitive decline is an associated comorbidity of apathy (e-13, e-43, e-53, e-75, e-114, e-241, e-424, e-248, e-264). Two studies also explicitly described overlapping mechanisms between apathy and depression, again offering a possible explanation as to why depression is an associated comorbidity of apathy 
(e-67, e-241). In six studies, the mechanisms of apathy in PD were directly linked to decision-making capacity and reward sensitivity $(9,10, \mathrm{e}-50, \mathrm{e}-129, \mathrm{e}-144, \mathrm{e}-11 \mathrm{~A})$. These studies suggest dopamine imbalances in those with apathy may limit an individual's sensitivity to reward and therefore hinder their decision-making capacity $(9,10$, e-50, e-129, e-144, e-11A).

\section{Description of overall management}

Overall management of apathy in PD was considered any information that aids treatment, and provides methods to lessen the burden felt by persons with PD and their caregivers. There is currently no guideline for managing apathy in PD. A key finding of this review is the negative effects apathy has on both persons with PD and their caregivers; in some studies apathy was reported as the most burdensome symptom in PD (e-26, e-35, e-148, e-177). The burden of managing apathy in PD was assessed by 28 studies (table 2; online supplementary table 3A). Five were specifically concerned with caregiver burden, concluding that managing apathy in PD increases the overall burden felt by the caregiver (47, e-40, e-149, $\mathrm{e}-177$, e-208). The most common conclusion in the remaining studies was that apathy has a negative effect on quality of life for persons with PD; with four studies concluding apathy is the most burdensome NMS in PD (e-26, e-35, e-148, e-177). One of the most common conclusions of all included studies was the need for future research on apathy to guide the development of a management plan, or more clear knowledge to provide to caregivers and persons with PD and apathy to ease the burden of managing this symptom.

\section{DISCUSSION}

This study identified 362 studies that contained information on the diagnosis, treatment or management of apathy in PD. Increased awareness of the non-motor symptoms in PD has resulted in increased research on this topic in the past decade; $78 \%$ of studies included within this review were published between 2010 and 2019. Despite this, apathy remains one of the most complex and least understood symptoms in PD. Those faced with apathy in PD currently have limited evidence-based options to manage this symptom.

\section{Diagnosis}

Our findings suggest making a diagnosis of apathy is a complex process, in part due to the variety of available screening tools. This study identified 20 different screening tools used for apathy in PD. The NPI and NPI-Q used by a total of $14 \%$ of included studies, was suggested for use to provide a broad assessment of apathy. Furthermore, the NPI-Q provides an even briefer assessment of apathy in PD and has not been validated for apathy case finding. ${ }^{13}$ The UPDRS Part 1 Item 4 (used in $10 \%$ of studies) may be suitable for initial screening, but is not recommended due to its limited ability to fully assess apathy (41, e-92). The AS and the LARS have been specifically developed and validated within PD populations and are recommended for use; these tools were used by $26 \%$ and $10 \%$ of studies, respectively. ${ }^{5}$ Therefore, of the included studies, only $36 \%$ applied a recommended apathy-screening tool. The use of non-recommended apathy-screening tools may bring into question the validity of the current body of literature, as not all tools used to identify apathy in the included studies are validated for use. The clinometric properties of the AS and LARS have also been assessed and prove to be valid measurements for the assessment of apathy in PD populations. ${ }^{15}$ Once gold standard criteria for apathy are developed, apathy screening tools will require further validation. ${ }^{513}$

Complexities associated with diagnosing apathy in PD may also be attributed to the variety of comorbidities, including depression and cognitive impairment, which may present with apathy. The range in prevalence of apathy was initially reported to be between $17 \%$ to $70 \%$ (24, e-172). A recent meta-analysis on apathy prevalence reported a pooled prevalence value of $40 \% .^{6}$ This estimate includes all persons with PD and apathy, regardless of the presence of key comorbidities. ${ }^{6}$ When accounting for only those with apathy, who had been screened and excluded if they had depression and/or cognitive impairment, the prevalence was $20 \%$; demonstrating how comorbidities may distort apathy prevalence estimates. ${ }^{6}$ What can be learnt from the above observations is that studies must clearly define pure apathy versus apathy that presents with depression, or cognitive impairment. Thus, the primary concern remains the development of gold standard criteria; which is then used to re-assess all available screening tools and considers their ability to identify apathy in the presence of common comorbidities. ${ }^{5}$ Validation against gold standard criteria will ensure that future apathy research accounts for the variety of ways apathy may present. Our scoping review also found inconsistent reports of apathy as a prodromal symptom of PD versus a symptom associated with motor symptom severity, duration of PD and older age. Improved screening measures will provide an opportunity to clarify the aforementioned relationships. The development of a measure for apathy that accounts for the variety of ways it may present has also been identified as a key area for future research on apathy in other neurodegenerative disorders such as $\mathrm{AD}$ and cognitive impairment, highlighting the importance of such research taking place in PD populations as well (e-263).

Further observations regarding diagnostic processes include, understanding discrepancies between caregiver and self-rated apathy screening tools. ${ }^{47-50}$ This may be due to the inherent nature of apathy, in that persons with PD and apathy have limited awareness of their behaviour, and therefore assess themselves as less apathetic (e-40, e-44). Cognitive decline that often presents with apathy may impair one's ability to accurately respond to selfrated tools (e-40, e-44). Thus, caregiver rated tools may provide a more accurate diagnosis, with one study 
identifying apathy diagnosed by the caregiver version of the NPI predicted the onset of PD dementia. ${ }^{12}$ Alternatively, as apathy was also found to be the most burdensome NMS in some studies, caregivers may over-report symptom severity due to the burden they feel because of the apathy (e-26, e-35, e-148, e-177). Once a gold standard definition is adopted within the literature, this observation should be taken into consideration to ensure both self and caregiver rated tools are validated. This will help address which version of these tools yields more dependable results, accounting for cognitive impairment.

\section{Treatment}

There were no conclusive findings regarding an effective treatment for apathy in PD. In many cases, where improvements in apathy were reported, it may be due to the treatment of the associated depression or cognitive impairment. ${ }^{16-20}{ }^{54}$ Future research must focus on large scale randomised trials, with sufficient follow-up time to account for the longer-term effectiveness of pharmacological and non-pharmacological interventions. The relationship between levodopa treatment and apathy symptoms was not extensively understood, and should also be a focus for future research in both de novo and non-de novo apathy populations. The application of a screening tool for apathy that is sensitive to improvements in apathetic behaviour is required, to better standardise how improvements in apathy are measured. ${ }^{15}$

While there were, limited studies investigating nonpharmacological interventions to treat apathy in $\mathrm{PD}$, exercise appears to improve apathy (e-204, e-62, e-111, e-133). Exercise as a treatment method also has the potential to aid in improving motor-symptoms in PD; a systematic review of 14 exercise-based RCT concluded exercise-based interventions improve physical functioning, strength, balance and gait. ${ }^{61}$ It will be important for future studies to identify the ideal form of exercise to improve apathy in PD, as the methods and interventions used in the five positive studies varied considerably.

\section{Gaps to an overall management approach}

As described above, overall management of apathy in PD is considered any information that will aid treatment, and provide interventions to lessen the burden of apathy in PD. The care provided to individuals with PD often is provided by family caregivers in the home, it is therefore important to provide them with a means to understand apathy as a symptom and care for these individual (e-149, e-177). This is especially true when considering that apathy was identified as being the most burdensome symptom by those with PD, and if care is provided from within the home this will in turn effect the burden felt by family caregivers (e-26, e-35, e-148, e-177). It is important when examining apathy, that caregivers are included as part of the study.

Overall, the largest hindrance to the development of an optimal approach to managing apathy in PD is the lack of gold standard criteria for apathy. The lack of gold standard criteria for apathy in turn prevents the accurate or uniform validation of measurement tools, and leading to variation measurement across studies. The lack of gold standard or clearly accurate tools in turn affects outcome measurement in intervention trials. While there were 362 studies included within this review, fewer than $50 \%$ of these studies used a recommended apathy-screening tool, which limits validity and power of studies designed to assess treatment methods for apathy. Without fundamental understandings of apathy and how it is best defined, we cannot develop a care pathway to aid in the overall management of apathy in PD. This in turn limits options that are available for healthcare practitioners, patients and caregivers regarding how to manage apathy in PD.

\section{Limitations}

Limitations of this research include the potential that we have missed important original literature on apathy in PD. This was mitigated by an extensive search of multiple databases, a large grey literature search, inclusion of all languages, searching references of included articles and ensuring duplicate review of all abstracts/ full-text. A thorough search of the grey literature was conducted, searching all relevant sources outlined by the CADTH grey literature tool. ${ }^{36}$ For practicality reasons, we chose not to include non-original or non-peer-reviewed research. While we may miss key conclusions drawn by such research, the references of such this literature were searched for all original, peer-reviewed citations for inclusion. We aimed to provide an entire scope of all original research on apathy in $\mathrm{PD}$, to allow for the synthesis of as broad scope of this literature as possible, we did not conduct a formal quality assessment of included studies. It is also important to note that anhedonia was not included as a final search term and that the explicit relationship between apathy and anhedonia was not investigated in this study.

\section{CONCLUSIONS}

This scoping review provides a comprehensive assessment of the literature on apathy in PD and demonstrates apathy and its presentation in PD are heterogeneous. Persons with PD may present with pure apathy, apathy with depression, apathy with cognitive impairment or apathy post-DBS. Overall, the largest gaps identified in this scoping review are the lack of a standardised apathy criteria and a validated, universal screening tool. There is also limited evidence on gold standard treatment for apathy in PD. Future directions should focus specifically on a clearer diagnostic process for apathy, including ensuring clinicians are aware of current apathy screening tools. Future research should also focus on assessing which treatments, both pharmacological and nonpharmacological are more effective to treat apathy in PD, as the primary outcome of interest. 
Author affiliations

${ }^{1}$ Department of Community Health Sciences, University of Calgary, Calgary, Alberta, Canada

${ }^{2}$ Cumming School of Medicine, University of Calgary, Calgary, Alberta, Canada

${ }^{3}$ Alberta Health Services, Calgary, Alberta, Canada

${ }^{4}$ Department of Medicine, University of Calgary, Calgary, Alberta, Canada

${ }^{5}$ Department of Psychiatry, University of Calgary, Calgary, Alberta, Canada

${ }^{6}$ Department of Clinical Neuroscience, University of Calgary, Calgary, Alberta,

Canada

${ }^{7}$ Hotchkiss Brain Institute, Calgary, Alberta, Canada

${ }^{8} 0^{\prime}$ Brien Institute for Public Health, Calgary, Alberta, Canada

Acknowledgements The authors would like to thank research librarian Zahra Premji, PhD, MLIS, and Diane Lorenzetti, PhD, MLS, for their assistance and input during the development of the search strategy and Monika Khoury-Dool for her assistance with acquiring project documentation. We would also like to thank the University of Calgary Brenda Strafford Foundation Chair in Geriatric Medicine, as having supported this research.

Contributors BSM was involved in study design, development of scoping review search, data collection, data analysis, data interpretation, figures and writing. SV was involved in updating the scoping review search, screening updated search abstracts and full-text, data collection, data analysis, data interpretation, figures and writing. Dr JH-L was involved with revisions to manuscript, analysis and interpretation of data. Dr ZI was involved with revisions to manuscript, analysis and interpretation of data. Dr TP was involved with revisions to manuscript, analysis and interpretation of data. Dr ZSG was involved with the development of the scoping review search, data verification, data interpretation, revisions to manuscript and study supervision

Funding The authors have not declared a specific grant for this research from any funding agency in the public, commercial or not-for-profit sectors.

Competing interests None declared.

Patient and public involvement Patients and/or the public were not involved in the design, or conduct, or reporting, or dissemination plans of this research.

Patient consent for publication Not required.

Provenance and peer review Not commissioned; externally peer reviewed.

Data availability statement All data relevant to the study are included in the article or uploaded as supplementary information.

Open access This is an open access article distributed in accordance with the Creative Commons Attribution Non Commercial (CC BY-NC 4.0) license, which permits others to distribute, remix, adapt, build upon this work non-commercially, and license their derivative works on different terms, provided the original work is properly cited, appropriate credit is given, any changes made indicated, and the use is non-commercial. See: http://creativecommons.org/licenses/by-nc/4.0/.

ORCID iDs

Bria Mele http://orcid.org/0000-0002-6931-5153

Tamara Pringsheim http://orcid.org/0000-0002-5057-9693

\section{REFERENCES}

1 Dujardin K, Sockeel P, Devos D, et al. Characteristics of apathy in Parkinson's disease. Mov Disord 2007;22:778-84.

2 Sinha N, Manohar S, Husain M. Impulsivity and apathy in Parkinson's disease. J Neuropsychol 2013;7:255-83.

3 Marin RS. Apathy: a neuropsychiatric syndrome. J Neuropsychiatry Clin Neurosci 1991;3:243-54.

4 Chase TN. Apathy in neuropsychiatric disease: diagnosis, pathophysiology, and treatment. Neurotox Res 2011;19:266-78.

5 Pagonabarraga J, Kulisevsky J, Strafella AP, et al. Apathy in Parkinson's disease: clinical features, neural substrates, diagnosis, and treatment. Lancet Neurol 2015;14:518-31.

6 den Brok MGHE, van Dalen JW, van Gool WA, et al. Apathy in Parkinson's disease: a systematic review and meta-analysis. Movement Disorders 2015;30:759-69.

7 Chaudhuri KR, Martinez-Martin P, Odin P, et al. Handbook of NonMotor Symptoms in Parkinson's Disease. Heidelberg: Springer Healthcare UK, 2011. http://link.springer.com/10.1007/978-1908517-60-9
8 Ziropadja L, Stefanova E, Petrovic M, et al. Apathy and depression in Parkinson's disease: the Belgrade PD study report. Parkinsonism Relat Disord 2012;18:339-42.

9 Buelow MT, Frakey LL, Grace J, et al. The contribution of apathy and increased learning trials to risky decision-making in Parkinson's disease. Arch Clin Neuropsychol 2014;29:100-9.

10 Lawrence AD, Goerendt IK, Brooks DJ. Apathy blunts neural response to money in Parkinson's disease. Soc Neurosci 2011;6:653-62.

11 Dujardin K, Sockeel P, Delliaux M, et al. Apathy may herald cognitive decline and dementia in Parkinson's disease. Mov Disord 2009:24:2391-7.

12 Fitts W, Weintraub D, Massimo L, et al. Caregiver report of apathy predicts dementia in Parkinson's disease. Parkinsonism Relat Disord 2015;21:992-5

13 Clarke DE, Ko JY, Kuhl EA, et al. Are the available apathy measures reliable and valid? A review of the psychometric evidence. $J$ Psychosom Res 2011;70:73-97.

14 Starkstein SE, Leentjens AFG. The nosological position of apathy in clinical practice. J Neurol Neurosurg Psychiatry 2008;79:1088-92.

15 Carrozzino D. Clinimetric approach to rating scales for the assessment of apathy in Parkinson's disease: a systematic review. Prog Neuropsychopharmacol Biol Psychiatry 2019;94:109641.

16 Devos D, Moreau C, Maltête D, et al. Rivastigmine in apathetic but dementia and depression-free patients with Parkinson's disease: a double-blind, placebo-controlled, randomised clinical trial. J Neurol Neurosurg Psychiatry 2014;85:668-74.

17 Moretti R, Caruso P, Dal Ben M. Rivastigmine as a Symptomatic Treatment for Apathy in Parkinson's Dementia Complex: New Aspects for This Riddle, 2017. Available: https://www.hindawi.com/ journals/pd/2017/6219851/ [Accessed 9 Feb 2018].

18 Bullock R, Cameron A. Rivastigmine for the treatment of dementia and visual hallucinations associated with Parkinson's disease: a case series. Curr Med Res Opin 2002;18:258-64.

19 Oh Y-S, Kim J-S, Lee PH. Effect of rivastigmine on behavioral and psychiatric symptoms of Parkinson's disease dementia. J Mov Disord 2015;8:98-102.

20 Litvinenko IV, Odinak MM, Mogil'naya VI, et al. Efficacy and safety of galantamine (Reminyl) for dementia in patients with Parkinson's disease (an open controlled trial). Neurosci Behav Physiol 2008:38:937-45.

21 Boyle PA, Malloy PF. Treating apathy in Alzheimer's disease. Dement Geriatr Cogn Disord 2004;17:91-9.

22 Kirsch-Darrow L, Marsiske M, Okun MS, et al. Apathy and depression: separate factors in Parkinson's disease. J Int Neuropsychol Soc 2011;17:1058-66.

23 Kirsch-Darrow L, Fernandez HH, Fernandez HF, et al. Dissociating apathy and depression in Parkinson disease. Neurology 2006:67:33-8.

24 Alzahrani H, Venneri A. Cognitive and neuroanatomical correlates of neuropsychiatric symptoms in Parkinson's disease: a systematic review. J Neurol Sci 2015;356:32-44.

25 Oguru M, Tachibana H, Toda K, et al. Apathy and depression in Parkinson disease. J Geriatr Psychiatry Neurol 2010;23:35-41.

26 Levy ML, Cummings JL, Fairbanks LA, et al. Apathy is not depression. J Neuropsychiatry Clin Neurosci 1998;10:314-9.

27 Luo XF, Zhang BL, Li JC, et al. Lateral habenula as a link between dopaminergic and serotonergic systems contributes to depressive symptoms in Parkinson's disease. Brain Res Bull 2015;110:40-6.

28 Levy R, Dubois B. Apathy and the functional anatomy of the prefrontal cortex-basal ganglia circuits. Cereb Cortex 2006:16:916-28.

29 Peters M, Godfrey C, Mclnerney P, et al. The Joanna Briggs Institute Reviewers' Manual 2015: Methodology for JBI Scoping Reviews, 2015. https://espace.library.uq.edu.au/view/UQ:371443

30 Mele BS. Understanding How to Care about Indifference: A Multistep Approach to Apathy in Parkinson's Disease. Open Sci Framew 2017.

31 Moher D, Shamseer L, Clarke M, et al. Preferred reporting items for systematic review and meta-analysis protocols (PRISMA-P) 2015 statement. Syst Rev 2015;4:1.

32 Arksey H, O'Malley L. Scoping studies: towards a methodological framework. Int J Soc Res Methodol 2005;8:19-32.

33 Levac D, Colquhoun H, O'Brien KK. Scoping studies: advancing the methodology. Implement Sci 2010;5:69.

34 Tricco AC, Lillie E, Zarin W, et al. PRISMA extension for scoping reviews (PRISMA-ScR): checklist and explanation. Ann Intern Med 2018;169:467-73.

35 Sampson M, McGowan J, Lefebvre C, et al. Press: peer review of electronic search strategies. OTT can agency drugs Technol health 2008. 
36 CADTH. Grey Matters: a practical tool for searching health-related grey literature | CADTH.ca[Internet]. Available: https://www.cadth.ca/ resources/finding-evidence/grey-matters [Accessed 12 Jun 2017]

37 Pham MT, Rajić A, Greig JD, et al. A scoping review of scoping reviews: advancing the approach and enhancing the consistency. Res Synth Methods 2014;5:371-85.

38 Daudt HML, van Mossel C, Scott SJ. Enhancing the scoping study methodology: a large, inter-professional team's experience with Arksey and O'Malley's framework. BMC Med Res Methodol 2013;13:48

39 Robert P, Onyike CU, Leentjens AFG, et al. Proposed diagnostic criteria for apathy in Alzheimer's disease and other neuropsychiatric disorders. Eur Psychiatry 2009;24:98-104.

40 Stella F, de Andrade LP, Garuffi M, et al. Validation of the Brazilian version of the apathy inventory. Int J Geriatr Psychiatry 2013;28:979-86.

41 Weintraut R, Karádi K LT, Kovács M, et al. Lille Apathy Rating Scale and MDS-UPDRS for Screening Apathy in Parkinson's Disease. J Park Dis 2016;6:257-65.

42 Cummings JL, Mega M, Gray K, et al. The neuropsychiatric inventory: comprehensive assessment of psychopathology in dementia. Neurology 1994;44:2308-14

43 Kaufer DI, Cummings JL, Ketchel P, et al. Validation of the $\mathrm{NPI}-\mathrm{Q}$, a brief clinical form of the neuropsychiatric inventory. $J$ Neuropsychiatry Clin Neurosci 2000;12:233-9.

44 Goetz CG, Tilley BC, Shaftman SR, et al. Movement disorder Society-sponsored revision of the unified Parkinson's disease rating scale (MDS-UPDRS): scale presentation and Clinimetric testing results. Mov Disord 2008;23:2129-70.

45 Starkstein SE, Mayberg HS, Preziosi TJ, et al. Reliability, validity, and clinical correlates of apathy in Parkinson's disease. $J$ Neuropsychiatry Clin Neurosci 1992;4:134-9.

46 Marin RS, Biedrzycki RC, Firinciogullari S. Reliability and validity of the apathy evaluation scale. Psychiatry Res 1991;38:143-62.

47 Camargo CHF, Serpa RA, Matnei T, et al. The perception of apathy by caregivers of patients with dementia in Parkinson's disease. Dement Neuropsychol 2016;10:339-43.

48 McKinlay A, Grace RC, Dalrymple-Alford JC, et al. Neuropsychiatric problems in Parkinson's disease: comparisons between self and caregiver report. Aging Ment Health 2008;12:647-53.

49 Schiehser DM, Liu L, Lessig SL, et al. Predictors of discrepancies in Parkinson's disease patient and caregiver ratings of apathy, disinhibition, and executive dysfunction before and after diagnosis. $J$ Int Neuropsychol Soc 2013;19:295-304.

50 Drijgers RL, Dujardin K, Reijnders JSAM, et al. Validation of diagnostic criteria for apathy in Parkinson's disease. Parkinsonism Relat Disord 2010;16:656-60.

51 Butterfield LC, Cimino CR, Salazar R, et al. The Parkinson's active living (pal) program. J Geriatr Psychiatry Neurol 2017;30:11-25.

52 Ray Chaudhuri K, Martinez-Martin P, Antonini A, et al. Rotigotine and specific non-motor symptoms of Parkinson's disease: post hoc analysis of recover. Parkinsonism Relat Disord 2013;19:660-5.

53 Drijgers RL, Verhey FRJ, Tissingh G, et al. The role of the dopaminergic system in mood, motivation and cognition in Parkinson's disease: a double blind randomized placebo-controlled experimental challenge with pramipexole and methylphenidate. $J$ Neurol Sci 2012;320:121-6.

54 Hauser RA, Slawek J, Barone P, et al. Evaluation of rotigotine transdermal patch for the treatment of apathy and motor symptoms in Parkinson's disease. BMC Neurol 2016;16:90.

55 Maillet A, Krack P, Lhommée E, et al. The prominent role of serotonergic degeneration in apathy, anxiety and depression in de novo Parkinson's disease. Brain 2016;139:2486-502.

56 Maruyama T. New treatment of depression in Parkinson's disease. Int J Psychiatry Clin Pract 2003;7 Suppl 1:25-7.

57 Zahodne LB, Bernal-Pacheco O, Bowers D, et al. Are selective serotonin reuptake inhibitors associated with greater apathy in Parkinson's disease? J Neuropsychiatry Clin Neurosci 2012;24:326-30.

58 Leentjens AFG, Koester J, Fruh B, et al. The effect of pramipexole on mood and motivational symptoms in Parkinson's disease: a meta-analysis of placebo-controlled studies. Clin Ther 2009;31:89-98.

59 Antonini A, Bauer L, Dohin E, et al. Effects of rotigotine transdermal patch in patients with Parkinson's disease presenting with nonmotor symptoms - results of a double-blind, randomized, placebocontrolled trial. Eur J Neurol 2015;22:1400-7.

60 Chung SJ, Asgharnejad M, Bauer L, et al. Evaluation of rotigotine transdermal patch for the treatment of depressive symptoms in patients with Parkinson's disease. Expert Opin Pharmacother 2016;17:1453-61.

61 Goodwin VA, Richards SH, Taylor RS, et al. The effectiveness of exercise interventions for people with Parkinson's disease: a systematic review and meta-analysis. Mov Disord 2008;23:631-40. 